\title{
BioSpotlight
}

\section{Closing the gap}

As sequencing costs continue to plummet, more high-quality draft genomes will be produced at a faster pace. While the raw data can be generated quickly, and the computational algorithms to assemble genomes continue to improve, what about closing gaps and checking for misassembled regions within those genome assemblies? It turns out that finishing remains a significant challenge when it comes to whole genome sequencing. In particular, closing gaps requires direct sequencing of PCR amplicons covering the gap regions and the assembly of those sequences into the genome assemblies. Sequencing of pooled gap-region PCR products using the single molecule sequencing system from Pacific Biosciences (PacBio) is more cost-effective than Sanger sequencing of individual PCR amplicons. However, while the PacBio system has the ability to sequence large size fragments in a single read (up to several kilobases in size), a challenge when pooling hundreds of PCR products for sequencing is the tendency for smaller products to load more efficiently into PacBio wells than larger ones, leading to skewing in the sequence distribution towards the smaller PCR fragments. In this issue of BioTechniques, researchers from Los Alamos National Laboratory (Los Alamos, New Mexico) describe how they have overcome this loading bias against larger PCR amplicons in pooled samples by adjusting the molar ratios of products based on their size and concentration. Eliminating this bias was key for the authors since the true value of the PacBio system lies in its longer read lengths, and the authors demonstrated this with their ability to close gaps of greater than $2.5 \mathrm{~kb}$ in a single round-a feat not possible using Sanger sequencing. In addition, gaps caused by hard stops (i.e. regions with strong secondary structure) are much more amenable to single molecule-based sequencing than other methodologies. A drawback with the PacBio system, however, is its high error rate, but the authors suggest that by increasing the accuracy of the amplicon consensus sequences through the use of additional sub-reads this issue can be largely overcome. Sequencing pools of gap-regions PCR products is a unique application of the single molecule sequencing technology that will definitely enhance the efficiency and speed of gap closure for draft genomes in the future.

\section{See "Improving genome assemblies by sequencing PCR} products with PacBio" on page 61.

\section{Resolving power}

In cell biology today, advances in resolution, along with the ability to reconstruct 3D images, have been crucial to visualizing cellular structures and deciphering biological processes. A major challenge in moving toward even higher resolution when imaging the insides of cells lies in the difficulty associated with imaging in the z-plane. Transmission electron microscopy (TEM) tomography enables acquisition of $3 \mathrm{D}$ biological datasets down to $3 \mathrm{~nm}$ resolution; however, TEM tomography is often limited to 0.5 micron thick sections which makes reconstruction of whole cells challenging. Another technique-focused ion beam scanning electon microscopy (FIB-SEM) that uses a focused beam of ions operating at low current for imaging while at higher currents the ion beam "mills" a sample - has enabled single nanometer resolution, but thus far not to the same level as TEM tomography. Reporting in this issue of BioTechniques, a team of researchers led by Kirk Czymmek at the University of Delaware, detail their optimization steps for FIB-SEM imaging that enables imaging and quantification of sub-cellular structures in single yeast cells at resolutions approaching $3 \mathrm{~nm}$ in the $\mathrm{x}^{-}, \mathrm{y}^{-}$, and even $\mathrm{z}$ - directions; the highest reported resolution using FIB-SEM to date. While Czymmek and

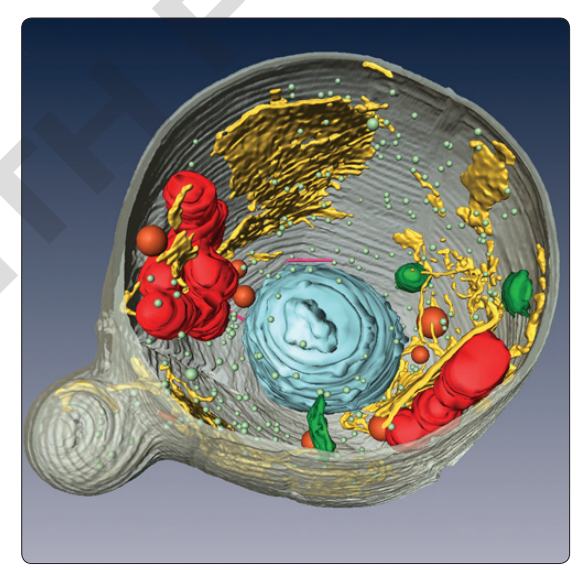

3D segmentation. Anisotropic FIB data set showed endoplasmic reticulum (ER; yellow), nucleus (light blue), cisternae (green), vesicles (light green), lipid droplets (orange), microtubules (pink), mitochondria (red) and cell wall (gray).

his colleagues made a significant effort to optimize the initial preparation steps prior to imaging to improve resolution, they also enhanced the electron collection procedure by altering the position of the electron detector to limit the number of electrons collected with large scattering angles or that originated from deep within samples. To demonstrate FIB-SEM capabilities using these optimized steps, the authors imaged a yeast cell down to $3 \mathrm{~nm}$ isotropic voxels, a six-fold reduction in $\mathrm{z}$-interval from previous FIB-SEM datasets and comparable to recent reports of $5 \mathrm{~nm}$ isotropic data from brain tissue. They were also able to calculate volume, volume percentage, and surface area of subcellular organelles including vacuoles and mitochondria. The modifications reported here by the authors will open the door to further exploration of the smallest structures and processes in eukaryotic cells.

See "High-resolution three-dimensional reconstruction of a whole yeast cell using focused-ion beam scanning electron microscopy" on page 41 .

Written by Nathan Blow, Ph.D. and Patrick Lo, Ph.D. [G]

BioTechniques 53:12 (July 2012)

doi $10.2144 / 000113883$

To purchase reprints of this article, contact:

biotechniques@fosterprinting.com 